\title{
Light aversion of normal and posterior neodecorticate rats
}

\author{
IAN Q. WHISHAW \\ University of Lethbridge, Lethbridge, Alberta, Canada T1K $3 M_{4}$
}

\begin{abstract}
The light aversion behavior of normal rats and rats with posterior neocortex lesions was examined in a two-choice brightness (card) discrimination task. By varying pretraining procedures, normal rats could be shown to have apparent light preferences, light aversion, or no preferences. Neither normal rats nor lesioned rats showed light avoidance tendencies in tasks in which pretraining biases were removed. The results indicate that the light avoidance tendencies shown by the normal or lesioned rats in free choice tasks are not reflected on card discrimination tasks.
\end{abstract}

A number of investigations have been interpreted as indicating that removal of part or all of visual neocortex in the rat leads to a reduction in light aversion tendencies (Altman, 1962; Bauer, 1968; Horel, Bettinger, Royce, \& Meyer, 1966; Krechevsky, 1936; Parker, Erickson, and Treichler, 1969). An extensive examination of the performance of normal and visual-system lesioned rats (Whishaw, 1974) has indicated that there is no difference in light avoidance tendencies in normal rats as compared to rats with posterior neocortex lesions. Other studies which have used lights as a discrimination stimulus in a two-choice acquisition test have suggested that procedural variables may differentially affect performance scores of normal and lesioned rats (Parker \& Treichler, 1973; Stratton, 1971; Stratton \& Edwards, 1971). The present experiments were designed to reexamine the performance of animals on a brightness discrimination task in which black or white cards are used as the discrimination stimulus. Horel et al. (1966) have suggested that error scores during acquisition reveal that lesioned rats show less aversion to the white card than normal rats. The present results suggest that procedural variables such as the method of pretraining can produce results which appear to indicate that rats have aversion tendencies, preferences, or no preferences for either black or white cards. Testing procedures which produced no preference in normal rats produced a similar lack of preference in rats with posterior neocortex lesions.

\section{METHODS}

\section{Subjects}

The experiments were conducted with 148 adult (250-350 g) female hooded rats of the Long-Evans strain. Forty-two rats received posterior neocortical lesions 2 weeks prior to testing. All of the animals were housed individually with free access to food and water.

This research was supported by grants from the Canadian National Research Council. Dr. J. D. Read sponsors this paper and takes full editorial responsibility for its content.

\section{Surgery and Histology}

Neocortical lesions were produced with the suction technique while animals were anesthetized with sodium pentobarbital. Circular portions of the skull $5 \mathrm{~mm}$ in diam were removed bilaterally $1.2 \mathrm{~mm}$ posterior to bregma. All neocortical tissue posterior to bregma and medial to the rhinal fissure was ablated. Lesions were identical in extent to those described by Bland and Cooper (1970). At the completion of the experiments, the animals were perfused under deep pentobarbital anesthesia and the brains were removed. Brains were photographed and then cut into 40-micron sections and stained with thionin for examination.

\section{Apparatus}

Three two-choice discrimination boxes with dimensions the same as those described by Thompson and Bryant (1955) were used. The boxes consisted of start, choice, and goal chambers. Entry into the boxes was gained via two $4 \times 4$ in. openings located at the end of two 5 -in. alleys. The start and choice chambers and the alleys had grid floors that could be independently electrified. Illumination was provided by an overhead bank of fluorescent lamps. The interior of one box was painted in flat black, one in flat white, and one in a gray intermediate to the black and white.

\section{Procedure}

Pretraining. Animals were placed in the apparatus in groups of four and allowed to explore for $5 \mathrm{~min}$. Each animal was then trained to run from start to goalbox to avoid a .8-mA dc footshock (resistance $100 \mathrm{~K}$ ). Over 15 trials, the animals were gradually trained to knock down pretraining doors which were first ajar and then fully closed and which were placed at the entrance to the goalbox.

Tests. Three separate tests were conducted: (1) Sixty-four normal animals were divided into groups of eight, pretrained with gray stimulus cards in either the gray, white, or black box. The different groups were then trained with either a black or white card as a positive stimulus, and the opposite color as a negative stimulus, within the respective training boxes. (2) Thirty-six rats, 18 normal, 18 posterior decorticate, were pretrained with gray cards in the gray box, divided into groups of nine and trained to approach and knock down either a black or a white card in the gray box. (3) Forty-eight rats, 24 normal, 24 lesioned, were pretrained in the gray box with gray cards and then divided into groups of six and then trained to run to either black or white cards in the black or white boxes.

Training. On the day following pretraining, the animals began discrimination training. Each rat received 10 trials/day with a 30 to 60 -sec intertrial interval. Entrance to the correct alley allowed an animal to knock down the stimulus card and gain entry to the goalbox. Footshock was given if the animal entered an incorrect alley, and the negative panel was locked so that entrance to the goal could not be obtained. If on a given trial an animal had not approached either of the two stimulus panels after $20 \mathrm{sec}$, it 
Figure 1. Representative examples of brains of posterior neodecorticate lesioned rats. Such lesions are accompanied by complete degeneration of the dorsal part of the lateral genlculate body (see Bland \& Cooper, 1970).

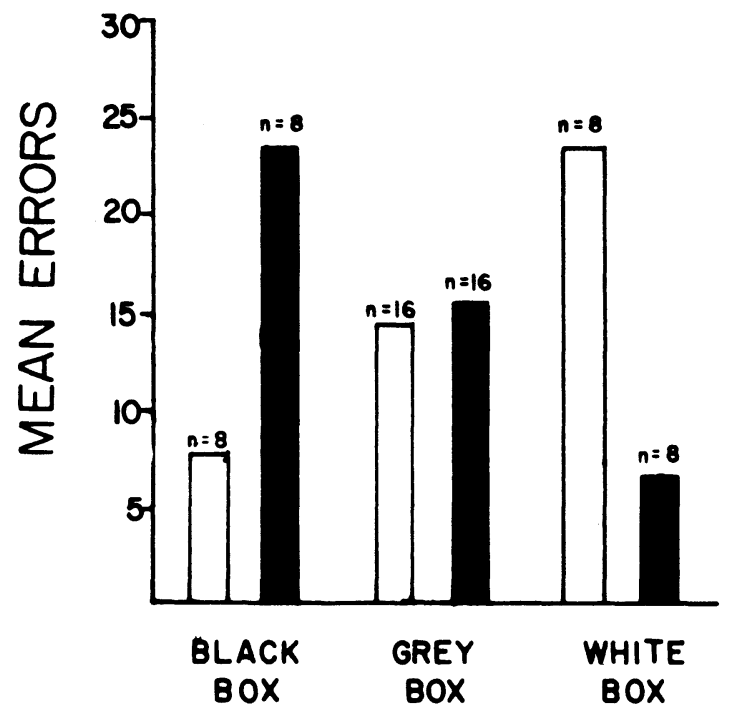

Figure 2. Mean error scores of normal rats on a black-white discrimination task in black, gray, and white boxes. Open bars, white card positive; solid bars, black card positive. Note superior performance to white card in black box, and black card in white box. Note equal number of errors to black and white in the grey box.

received footshock. Each animal was trained to a criteria of 18 out of 20 correct responses over 2 consecutive days.

\section{RESULTS AND DISCUSSION}

Examples of the extent of the posterior aspirations is shown in Figure 1. In all cases, the posterior neocortex was successfully removed. Examination of stained sections showed that the degeneration in the lateral geniculate body was comparable to that previously reported for lesions of similar extent (Bland \& Cooper, 1970).

A summary of the results of the first experiment is shown in Figure 2. There was no significant card effect across the three boxes, however the Card by Box interaction was highly significant $(\mathrm{F}=379, \mathrm{df}=1 / 28$, $p<.001)$. The entire interaction effect was due to the low error scores made to the contrasted cards in the black and white boxes $(p<.001, t$ tests $)$. The results show that in the gray box there is no evidence of brightness preference in performance but that apparent preference for white appears in the black box and apparent preference for black appears in the white box. The simplest interpretation of the results is that the use of gray cards as pretraining stimuli in the black and white boxes cues the animals to approach the cards contrasted against the background in the discrimination tests. Since this cuing effect does not occur in the gray box, the results suggest that the gray box is an appropriate situation to determine if there are preference changes after posterior neodecortication.

Figure 3 summarizes the results of the second experiment. The error scores of control rats to black and white cards were the same, confirming the results of the first experiment. The performance of the lesioned rats was inferior to that of control animals, but error scores and acquisition rates were not significantly different to white as compared to black cards $(p<.05)$. The results can be interpreted as showing that light aversion tendencies are not a significant factor in error scores to card stimuli in a brightness discrimination task for either normal or posterior neodecorticate rats.

The results of the third experiment are summarized in Figure 4. The results show that pretraining animals in the gray box with gray cards removes the cuing effect obtained with pretraining animals in black and white boxes. Both normal and lesioned rats pretrained in the gray box made equivalent error scores to black and white cards in the black and white boxes. There was a slight tendency for animals with lesions to do

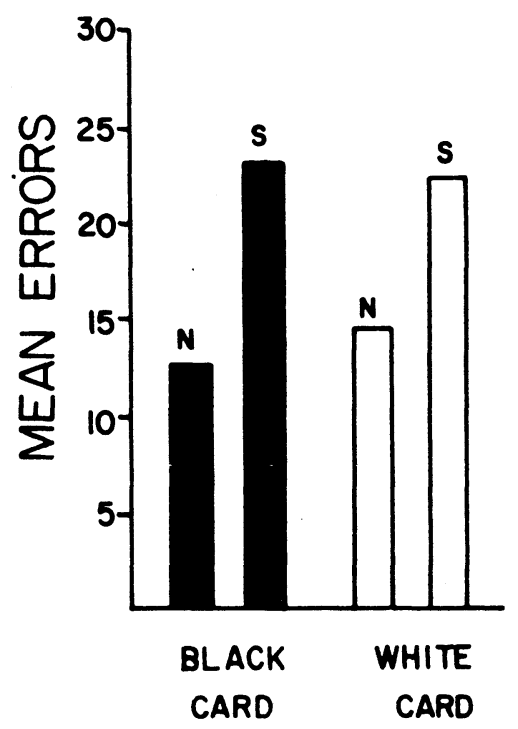

Figure 3. Mean error scores of normal (N) and posterior neodecorticate (striate, S) rats to black and white cards in the gray training box. Note lack of stimulus preference in normal and lesioned rats but overall superior performance of normal rats $(n=9$ in each group). 


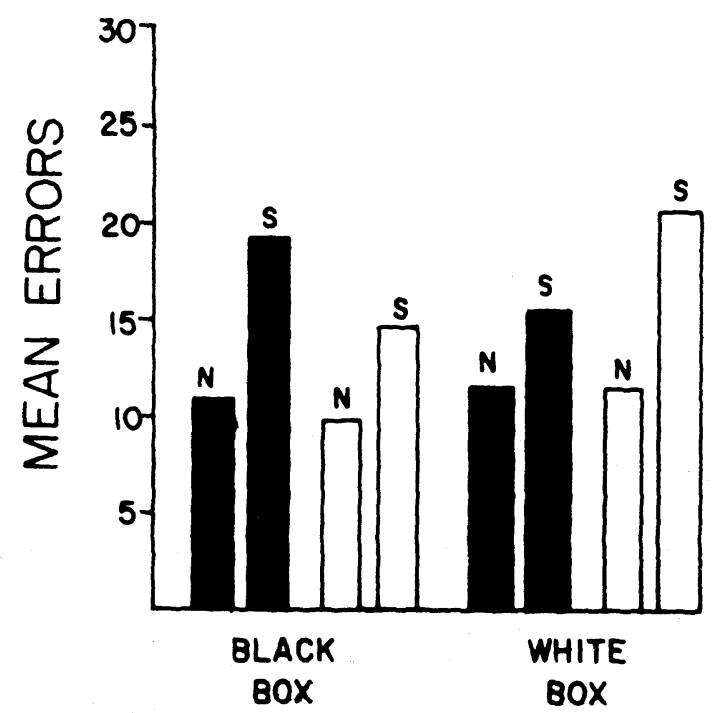

Figure 4. Mean error scores to black (solid bars) and white (open bars) cards by normal $(N)$ and posterior neocortex (striate, $S$ ) rats. Note: All rats were pretrained in a gray box. Error scores by both groups to both cards were equivalent but performance of normal rats was superior to performance of lesioned animals. $(n=6$ in each group).

somewhat better to the contrasted stimulus, but this effect was not significant ( $p>.05)$. Also, the tendency was apparent for both black and white cards and so does not reveal evidence for operation of preference factors. The results of the third experiment also confirmed the results of the second experiment in that performance of lesioned rats was inferior to control rats on all of the tasks.

In summary, the results of the experiments reveal that training procedures can determine performance levels in brightness discrimination tasks so that animals can appear to have light aversion tendencies, light preference tendencies, or no preferences. When care is taken to remove bias in training and testing, neither normal rats nor posterior decorticate rats show brightness preferences. The results suggest that care should be taken to evaluate all factors which might influence performance in discrimination tasks before conclusions concerning the operation of "light aversion tendencies" are evoked to explain performance levels.

\section{REFERENCES}

Altman, J. Effects of lesions in central nervous visual structures on light aversion in rats. American Journal of Physiology, 1962. 202. $1208-1210$

BAUER, J. H. Visual and nonvisual behavior of the rat after early and late posterior neocortical lesions. Doctoral dissertation, Faculty of Graduate Studies and Research, University of Manitoba, 1968.

Bland, B. H., \& COOPER, R. M. Experience and vision of the posterior neodecorticate rat. Physiology and Behavior, 1970. 5. 211-214.

Horel, J. A., Bettinger, L. A., Royce, G. J., \& Meyer, R. D. Role of the neocortex in the learning and relearning of two visual habits by the rat. Journal of Comparative and Phsyiological Psychology, 1966, 61, 66-78.

KRECHEVSKY. I. Brain mechanisms and brightness discrimination learning. Journal of Comparative Psychology, 1936, 21, 405-445.

Parker, T. D., Erickson, C. K., \& Treichler, F. R. The influence of brightness preferences on the assessment of cortical lesion deficits in rats. Journal of Comparative and Physiological Psychology, 1969, 67, 240-244.

Parker, T. D., \& Treichler, F. R. The influence of restricted posterior neodecortication on brightness preference and brightness discrimination performance of rats. Physiology and Behavior, 1973, 10, 473-478.

Stratton, L. O. Effects of dark preference on attention to the positive cue in posterior neodecorticate albino rats. Psychonomic Science, 1971, 24, 105-106.

Stratton, L. O.. \& EDwards, S. Preference and sensory factors in brightness discrimination learning of posterior neodecorticate albino rats. Psychonomic Science, 1971, 24, 9-10.

Thompson, R.. \& Bryant, H. J. Memory as affected by the relevant receptor. Psychological Reports, 1955, 1, 393-400.

Whishaw, I. Q. Light avoidance in normal rats and rats with primary visual system lesions. Physiological Psychology, 1974. 2. 143-147.

(Received for publication September 29, 1975.) 